\title{
Cancer did not stay home for COVID-19
}

\author{
Ross L. Levine ${ }^{1,2}$ \\ ${ }^{1}$ Human Oncology and Pathogenesis Program, ${ }^{2}$ Department of Medicine, Memorial Sloan Kettering Cancer Center \\ Email: leviner@mskcc.org
}

The COVID-19 pandemic has presented unprecedented challenges to society, to the health care system, and to academic medicine as a whole. It has been humbling to see the impact on every aspect of our society, including the biomedical research enterprise, of a highly contagious infectious disease which lacks definitive therapies/vaccines. Moreover, COVID-19 has presented new research and clinical questions that are being addressed at extraordinary speed, with a collaborative spirit, transparency and "can do" approach which speaks to the power of the scientific community. As a cancer doctor and researcher, I have seen countless examples of my colleagues providing front-line clinical care and cancer scientists using their expertise to solve critical questions related to COVID-19. Despite the tragedy unfolding before us, there is much to be heartened about with respect to how our community has responded. However, the pandemic has created many headwinds for the oncology community, with the potential for long-term consequences unless we redouble our efforts focused on cancer medicine and cancer research.

It is hard to accurately delineate the impact of COVID-19 on cancer care, and on the clinical oncology community. Cancer patients require frequent visits to their care provider for evaluation and for cancer therapy, and most cancer centers have seen a marked decrease in patient visits and in treatment volume during the height of the pandemic. Although in many cases clinicians have shown tremendous insight and flexibility, including the rapid adoption of telemedicine and thoughtful changes in therapeutic dosing and schedule to limit in-person infusion visits, the impact of limited access to cancer care on cancer outcomes has been substantive and will continue to be felt as the pandemic continues. Moreover, recent studies have shown that cancer patients who develop COVID-19 have significantly worse outcomes than COVID-19 patients without cancer. $(1,2)$ This has been particularly notable in patients with hematologic malignancies, however the adverse impact of COVID-19 in patients with active malignancies was seen across a broad spectrum of cancer types. Moreover, early data suggest that the rate of age-appropriate cancer screening, including mammography and colonoscopy, has dropped precipitously during the pandemic, and it is not yet clear when this will return to pre-pandemic levels. COVID-19 has been an existential health crisis for society, and nowhere has it been felt more directly than in the cancer community.

Cancer care models, staffing, and training have also been disrupted, with both short- and longer-term implications. Access to specialists, especially local expertise, for patients with rare cancers, has presented a challenge, mitigated in part by the temporary approval of telemedicine consults across state lines. Cancer-specific clinical providers, alongside all health care workers, have been asked to care for COVID-19 patients, including in ICUs and general medical wards, reducing staffing for cancer-specific delivery teams. This has been particularly apparent for clinical fellows and other trainees, who have gone above and beyond to contribute to the care of COVID-19 patients at the expense of their clinical and research training in cancer medicine/science. Moreover, accrual to clinical trials has dropped during the pandemic, such that the pace of clinical/translational cancer science has slowed down considerably.

In addition to the direct impact on cancer medicine in the clinical context, we have seen an equally substantive and concerning impact on discovery science and laboratory-based cancer research. Many non-profit foundations that support scientific research, including high-risk, high-reward ideas and early career investigators, have seen a reduction in philanthropic support and resultant decrease in grants. Moreover, the financial landscape at academic institutions has been adversely impacted by reduced health care revenue and philanthropy, which has reduced the ability of academic institutions to invest in new faculty hires and in programmatic initiatives. Fortunately, funding from the National Institutes of Health (NIH) for cancer research, and for biomedical research writ large, has not been impacted, and NIH funding for COVID-19 science has not come at the expense of support for cancer science. The commensurate reduction in grant support and institutional resources is already having a broad impact on the cancer biology field. Institutions have reduced or frozen faculty recruiting, such that there are a large set of trainees with reduced or absent job prospects in the foreseeable future. Reduced foundation funding has a disparate impact on trainees and early career scientists and will reduce the ability of the best and brightest to take scientific risks and broaden their inquiries at a pivotal stage in their career. Moreover, the lack of scientific conferences reduces critical opportunities for young investigators to present and promote their work and to establish themselves in the cancer research 
community. In sum, we are on the cusp of seeing a "lost generation" of scientists have their careers irreparably impacted. We risk losing the best and brightest in the cancer field at a time when there has never been greater capacity for translating scientific insights to benefit for cancer patients.

We therefore must advocate for collective efforts to support and preserve the cancer medicine/biology field. We need to advocate for continued, increased funding for cancer science, and for the basic science which informs fundamental mechanisms of disease including malignant transformation. We need increased support for early career investigators and trainees; they are our future and the engine for continued scientific impact. In the context of COVID-19, the cancer research field has already contributed in many ways to our collective efforts during the pandemic, including the role of pathologic inflammation, hematologic alterations, and the complex interplay between malignancy, cancer therapy, and COVID-19 disease. Cancer biology has always paved the way for biomedical innovation and improved human health, with broad, substantive impact. Cancer did not stay home during COVID-19, and we cannot stay home when the cancer field needs our collective efforts.

\section{REFERENCES AND NOTES}

1. V. Mehta, S. Goel, R. Kabarriti, D. Cole, M. Goldfinger, A. Acuna-Villaorduna, K. Pradhan, R. Thota, S. Reissman, J. A. Sparano, B. A. Gartrell, R. V. Smith, N. Ohri, M. Garg, A. D. Racine, S. Kalnicki, R. Perez-Soler, B. Halmos, A. Verma, Case Fatality Rate of Cancer Patients with COVID-19 in a New York Hospital System. Cancer Discov. 10, 935-941 (2020). doi:10.1158/2159-8290.CD-20-0516 Medline

2. M. Dai, D. Liu, M. Liu, F. Zhou, G. Li, Z. Chen, Z. Zhang, H. You, M. Wu, Q. Zheng, Y. Xiong, H. Xiong, C. Wang, C. Chen, F. Xiong, Y. Zhang, Y. Peng, S. Ge, B. Zhen, T. Yu, L. Wang, H. Wang, Y. Liu, Y. Chen, J. Mei, X. Gao, Z. Li, L. Gan, C. He, Z. Li, Y. Shi, Y. Qi, J. Yang, D. G. Tenen, L. Chai, L. A. Mucci, M. Santillana, H. Cai, Patients with Cancer Appear More Vulnerable to SARS-CoV-2: A Multicenter Study during the COVID-19 Outbreak. Cancer Discov. 10, 783-791 (2020). Medline

\section{ACKNOWLEDGMENTS}

Studies supported by Memorial Sloan Kettering core facilities were supported in part by Memorial Sloan Kettering Cancer Center Support Grant/Core Grant P30 CA008748. R.L.L. is on the supervisory board of Qiagen and is a scientific advisor to Loxo/Lilly, Zentalis, Imago, Mana Therapeutics, Auron, Ajax, Syndax, C4 Therapeutics and Isoplexis. He receives research support from Prelude Therapeutics and has consulted for Celgene and Gilead.

Published First Release 17 July 2020

10.1126/sciadv.abd7487 